\title{
Estrategias para la implementación efectiva de la cadena de frío en la región de Azuero
}

\section{Strategies for the effective implementation of the cold chain in the Azuero region}

\author{
Lilibeth Madrid $^{1^{*}}$, Humberto Álvarez ${ }^{2}$ \\ ${ }^{1}$ Licenciatura en Ingeniería Industrial, Centro Regional de Azuero, Universidad Tecnológica de Panamá \\ ${ }^{2}$ Facultad de Ingeniería Industrial, Campus Víctor Levi Sasso, Universidad Tecnológica de Panamá,
}

\begin{abstract}
Resumen En esta investigación se realiza un análisis del sistema de cadena de frío, específicamente del Centro de Manejo Post Cosecha El Ejido, ubicado en la provincia de Los Santos, con el objetivo de proponer las estrategias que contribuyan con su implementación. En la primera fase se realizó la caracterización y análisis de cada uno de los componentes del sistema: centro de manejo post cosecha, transporte refrigerado y mercados públicos, que permitió identificar que el centro post cosecha solo tiene una efectividad del $11.9 \%$ y que el sistema carece de la totalidad de los equipos necesarios para mantener la cadena de frío. En la segunda fase se analizaron las prácticas post cosecha y de comercialización del productor de la región, así como su nivel de conocimiento sobre el tema estudiado, reflejando que en la región se ubican pequeños productores, con poca capacidad de gestión y planificación, lo que afecta directamente al centro post cosecha. Se identificó que un gran porcentaje de los productores están interesados en conocer los beneficios del sistema e implementarlo.
\end{abstract}

Palabras clave Agro logística, cadena de frío, hortofrutícola.

\begin{abstract}
In this document an analysis of the cold chain system is performed, specifically of the El Ejido Post - Harvest Management Center located in the Province of Los Santos, with the objective of proposing strategies that contribute to its implementation. In the first phase, the characterization and analysis of each of the components of the system was carried out: postharvest management center, refrigerated transport and public markets, which allowed identifying that the post-harvest center only has an effectiveness of $11.9 \%$ and that the system lacks all the necessary equipment to maintain the cold chain. In the second phase the post - harvest and marketing practices of the producers in the region were analyzed, as well as their level of knowledge on the subject in question, reflecting that there are small producers in the region with little management and planning capacity, which directly affects the post-harvest center. In the same way, it was identified that a large percentage of producers are interested in knowing the benefits of the system and implementing it.
\end{abstract}

Keywords Agro-logistic, cold chain, fruit and vegetable.

* Corresponding author: lilibeth.madrid@utp.ac.pa

\section{Introducción}

El estudio muestra el análisis del estado actual del sistema integral de la cadena de frío, principalmente del centro de manejo post cosecha de El Ejido, provincia de Los Santos, República de Panamá. El objetivo principal es el diseño de estrategias que incrementen el nivel de utilización del sistema y coadyuve al mejoramiento de las prácticas utilizadas por los productores de la región azuerence.

En el año 2018, solo el $2 \%$ de los productores que se pretendían beneficiar con el centro de post cosecha El Ejido, habían hecho uso del beneficio, que deja en evidencia la existencia de una infraestructura que no es utilizada y la necesidad de crear estrategias que promuevan un precio justo por los productos. Entre otros productores, el uso de esta plataforma logística, provoca un impacto positivo en el desarrollo económico y social de la región mediante el incremento del ingreso para el productor; el aprovechamiento de los recursos existentes; la reducción de la merma y las posibilidades

El estudio se divide en dos fases: El análisis del sistema y la postura del productor frente al propósito de identificar todos aquellos factores influyentes en el bajo nivel de implementación.

Inicialmente el documento contiene una breve descripción teórica sobre la cadena de frío, sus objetivos e importancia. Seguidamente se detallan las generalidades de la investigación: antecedentes, identificación del problema, justificación y el 
objetivo principal. En los puntos siguientes se explica la metodología utilizada y el resultado del análisis realizado a los componentes del sistema y a los productores de la región. Finalmente, se presentan las estrategias que se sustentan en los resultados del análisis realizado y que serán la base para el desarrollo de un plan de acción o mapa de ruta que señale a los actores los caminos adecuados para lograr la permanencia y la sostenibilidad de la cadena y que conduzcan el desarrollo de las actividades esta, hacia el logro de sus objetivos.

\section{Cadena de frío: objetivo e importancia}

Es el conjunto de procesos logísticos que se realizan bajo temperatura y humedad controlada, de acuerdo a las características de cada producto, desde el momento que se inicia la producción hasta que llega al consumidor final.

No solo es una serie ininterrumpida de instalaciones, actividades de almacenamiento y distribución en frío, especialmente para alimentos perecederos, dentro de condiciones de temperatura controlada, que requiere de infraestructuras y tecnologías, sino que requiere de la coordinación entre las partes interesadas, personal técnico capacitado, información adecuada y una buena gestión operativa [1].

Su principal objetivo es conservar y mantener los productos perecederos en los rangos de temperatura que el producto requiere para conservar sus propiedades, mediante el control de las condiciones que la pueden alterar (temperatura, humedad, ventilación, radiaciones y la calidad del envase) [2].

La importancia del sistema logístico de cadena de frío radica principalmente en que retrasa la degradación del alimento y de sus propiedades sensoriales, lo que evita la formación de microrganismos que dañan el producto. Mediante su uso se obtiene una serie de beneficios: reduce la merma, aumenta el tiempo de vida del producto, disminuye los riegos de la salud poblacional, reduce la volatilidad en el precio de los alimentos, facilita el proceso de comercialización y genera excedentes exportables.

Es importante resaltar que existe una serie de factores claves que influyen en el éxito de la cadena de frío y que están determinados por el impacto y su influencia; la ausencia o falla de tan solo uno de ellos, repercute negativamente en la conservación del producto. Estos factores son los siguientes: buenas prácticas de cosecha y post cosecha, trasporte, almacenamiento, distribución y venta en frío, almacenamiento en frío por los consumidores [3].

\section{Generalidades}

\subsection{Antecedentes}

Panamá es uno de los principales centros multimodales del mundo. Sin embargo, presenta carencia como plataforma logística y de servicios para alimentos perecederos. Para hacerle frente al estatus tradicional en el manejo de estos productos, se conceptualizó en el año 2009 , un proyecto que establecía la instalación de un sistema conocido como cadena de frío. En ese sentido, se creó la Secretaria de Cadena de Frío, que a partir del año 2013, pasó a ser Mercados Nacionales de la Cadena de Frío, S.A.

No fue hasta el año 2014, que el proyecto del sistema integral de cadena de frío agrícola se cristaliza y se convierte en uno de los más importantes desarrollados en Panamá. Un proyecto ambicioso con el objetivo de reducir la merma, reforzar las condiciones fitosanitarias, mejorar la calidad de los alimentos, generar excedente exportable y ofrecer a los pequeños y medianos productores un medio que facilite $y$ otorgue transparencia en la comercialización de productos perecederos.

La plataforma logística está orientada al manejo de 24 productos hortícolas, los cuales fueron seleccionados bajo los parámetros de volumen de producción, nivel de consumo y forma de comercialización [4].

$\begin{array}{lll}\text { 1. Ají Dulce } & \text { 9. Habichuela corta } & \text { 17. Chayote } \\ \text { 2. Pimentón } & \text { 10. Habichuela larga } & \text { 18. Coliflor } \\ \text { 3. Apio } & \text { 11. Lechuga } & \text { 19. Culantro } \\ \text { 4. Berro } & \text { 12. Mostaza } & \text { 20. Tomate de mesa } \\ \text { 5. Brócoli } & \text { 13. Papa } & \text { 21. Tomate industrial } \\ \text { 6. Cebolla } & \text { 14. Perejil } & \text { 22. Zanahoria } \\ \text { 7. Cebollina } & \text { 15. Pepino } & \text { 23. Espinaca } \\ \text { 8. Cilantro } & \text { 16. Remolacha } & \text { 24.Repollo }\end{array}$

El sistema se basa en una red de cuatro plantas de acopio ubicadas estratégicamente en las principales zonas de producción, tres (3) en la provincia de Chiriquí (Cerro Punta, Dolega y Volcán), una (1) en la provincia de Los Santos (El Ejido) y ocho (8) mercados públicos.

El centro post cosecha en la región de Azuero ubicado estratégicamente en el corregimiento de El Ejido, provincia de Los Santos, fue diseñado para realizar las operaciones de lavado, secado, parafinado, clasificación y selección de tomate, ají, pimentón, chayote y pepino, al igual que la reducción de la temperatura en cámaras de aire forzado con humedad controlada y almacenaje temporal de tránsito, con capacidad para procesar un volumen diario de 11,482 kilogramos de productos.

Datos proporcionados por los Mercados Nacionales de la Cadena de Frío S.A. revelan que para diciembre del 2017, en el centro del El Ejido, solo se recibieron 535 kilogramos de productos, sumado al hecho (según declaraciones del gerente del centro), que en ocasiones se han adaptado las instalaciones a la recepción de alimentos que no forman parte de los 24 rubros que se mencionan anteriormente, como es el caso del ñame, y en otras, existen días que no se recibe ningún producto. Esto deja en evidencia la existencia de un beneficio técnico que no es utilizado o aprovechado por el productor de la región.

En cuanto al mercado público del distrito de Chitré, provincia de Herrera, el cual forma parte de la plataforma logística y fue diseñado para garantizar el funcionamiento de 
la cadena de frío, no ha iniciado operaciones. Se esperaba su funcionamiento en junio del 2019.

\subsection{Problema de investigación}

Planteada la situación, queda en evidencia la existencia de una plataforma logística que no ha sido utilizada de manera eficiente; problemática que es atribuida a dos posibles razones: la primera, uso de la cadena de frío y la segunda, las deficiencias en los mecanismos de operación establecidos por la Secretaría de Cadena de Frío.

El estudio desarrollado se enfoca en atención a dos vertientes; una desde la perspectiva del productor y la otra, vista con relación al sistema de la cadena de frío, debido a la escasa información existente y consultada, revela deficiencias en ambas vías.

\subsection{Justificación}

En Panamá tan solo en el antiguo mercado agrícola de abastos se enfrentaban pérdidas de 136, 876, 000,000 de dólares debido a los 25,000 kilogramos de mermas que se registraban diariamente, principalmente en productos como los vegetales.

De acuerdo con los datos de la Asociación de Productores y Comerciantes Agrícolas del Mercado Central de Panamá, gran parte de los alimentos vendidos en el mercado interno derivaban en significativas pérdidas, las mermas en vegetales que alcanzaban el $40 \%$ y el $15 \%$ en frutas.

El proyecto de un sistema logístico de cadena de frío es una alternativa para contrarrestar el deterioro de productos. Su uso adecuado aportaría grandes beneficios a los productores, reduciendo las pérdidas ocasionadas por deficiencias en el manejo, almacenamiento y transporte, que reflejan sus beneficios en la calidad de los productos, disminución de mermas y el aumento de sus ingresos.

La estructura actual del sistema integral de cadena de frío constituye una inversión significativa y un avance fundamental en cuanto a mejorar la capacidad y eficiencia del agro, para direccionar los esfuerzos hacia su ejecución efectiva se convierte en prioridad para los sectores involucrados en materia, que buscan alcanzar su máximo beneficio y aportar al eslabón económico y social del país.

\subsection{Objetivo}

Diseñar y proponer estrategias que fortalezcan la implementación efectiva del sistema integral de la cadena de frío (centro de manejo post cosecha en la región de Azuero).

\section{Metodología}

\subsection{Tipo de investigación}

La investigación desarrollada es de carácter descriptivo explicativo; pues se caracteriza, reseña e identifican los factores influyentes en la implementación efectiva de la cadena de frío, y proporciona en un sentido completo el entendimiento del entorno de esta cadena (centro de manejo post cosecha en la región de Azuero).

\subsection{Procedimiento}

La primera etapa del proyecto consistió en una amplia revisión documental que aportó las bases teóricas para el desarrollo del estudio.

El trabajo de campo para el desarrollo de la investigación se realizó en dos fases:

En la primera fase se realizaron visitas al centro de post cosecha y Merca Panamá, con la finalidad de analizar los procesos que el productor debe seguir al momento de utilizar el sistema, además de estudiar los perfiles técnicos (equipos disponibles) y logísticos (sistemas de almacenamiento, vehículos de transporte), que permitieron la caracterización y evaluación de los aspectos relacionados al problema en cuestión desde el punto de vista de la cadena de frío.

En la segunda fase se realizó una encuesta estructurada con preguntas abiertas y cerradas a los productores de la región, con la finalidad de describir y analizar sus prácticas de transporte y comercialización, determinar el nivel de conocimiento sobre el tema y las actitudes tomadas frente a esta situación, así como de la obtención de información relevante que contribuyó con el desarrollo de la investigación.

Los resultados del análisis obtenido en ambas fases permitieron diseñar las estrategias que contribuirán con la utilización de la cadena de frío por parte del productor de la región.

\subsection{Cálculo de población y muestra para la aplicación de la encuesta}

La población estuvo compuesta por 207 productores inscritos en el Ministerio de Desarrollo Agropecuario (MIDA), cuyos rubros estaban dentro de los 24 seleccionados para utilizar el centro de manejo post cosecha y de los cuales 142 productores pertenecen a la provincia de Los Santos y el resto65) a la provincia de Herrera.

Si consideramos que la variable objeto de estudio es de tipo cuantitativa discreta (cantidad de productores) y la población es conocida (finita), se manejó la siguiente ecuación para determinar el tamaño de la muestra (1).

$$
n \geq \frac{Z^{2} N p q}{(N-1) e^{2}+Z^{2} p q}
$$

Donde:

$\mathrm{n}=$ tamaño de muestra

$\mathrm{N}=207$ productores

$\mathrm{Z}=1.96$, para un nivel de confianza del $95 \%$

$\mathrm{p}=$ probabilidad de éxito $50 \%$

$\mathrm{q}=$ probabilidad de fracaso $50 \%$

$\mathrm{e}=5 \%$, error estándar de estudio 
Al aplicar la fórmula (1), se determinó una muestra necesaria de 135 productores, suficientes para garantizar la confiabilidad y precisión deseada en los resultados. En cuanto a la selección de la muestra, se utilizó un muestreo de tipo estratificado para garantizar la presencia de todos los subgrupos claves dentro de la muestra. La división de los estratos se realizó primero por provincia, luego por región, rubro y finalmente se utilizó muestreo aleatorio para determinar la unidad muestral, en donde cada uno de los productores tenía la misma probabilidad de ser seleccionado.

\section{Resultados fase 1: Análisis de los componentes del sistema}

El Sistema Integral de Cadena de Frío de Panamá se compone de tres elementos: centros de manejo post cosecha (CMPC), flota de camiones refrigerados (FCR) y mercados públicos nacionales (MPN). En los siguientes puntos se presenta el resultado del análisis realizado a cada uno de estos componentes.

\subsection{Centro de Manejo Post Cosecha (CMPC) - El Ejido}

Son instalaciones de primer nivel diseñadas para el acopio, procesamiento, almacenaje y despacho de productos hortícolas frescos, operado por personal idóneo, con la finalidad de prolongar su vida útil. Su objetivo principal es servir de apoyo al productor en las operaciones de recepción, procesamiento y despacho de los rubros seleccionados, manteniendo las condiciones adecuadas de temperatura y humedad, reduciendo la merma al aumentar el tiempo de vida de los productos y mejorando su inocuidad.

\subsubsection{Capacidad y nivel de utilización}

El centro de manejo post cosecha El Ejido, objeto de este estudio, fue diseñado para procesar una capacidad anual de 4 , 133,442 kilogramos de productos y con estimaciones de beneficiar a 581 productores de la región [4]. Proyectando utilizar el $40 \%$ de su capacidad instalada para el primer año de operación (2014) y se espera alcanzar en un periodo de 12 años, la utilización del 93\% de esta capacidad instalada, con un crecimiento del $8 \%$ anual respecto al año anterior.

Sin embargo, el volumen de producto procesado en el CMPC para los años 2017 y 2018, mostrado en la tabla 1, no llegan a las cantidades esperadas, lo que refleja su baja tasa de utilización [5], [6].

Tabla 1. Volumen procesado esperado vs real
\begin{tabular}{|c|c|c|}
\hline Año & $\begin{array}{c}\text { Volumen } \\
\text { Esperado }(\mathbf{k g})\end{array}$ & $\begin{array}{c}\text { Volumen } \\
\text { Real }(\mathbf{k g})\end{array}$ \\
\hline 2017 & $2,082,778.6$ & $447,580.0$ \\
\hline 2018 & $2,249,400.9$ & $266,917.8$ \\
\hline
\end{tabular}

En el año 2017, la tasa de utilización esperada era de 50\% y la alcanzada fue de $11 \%$ (39\% no logrado); mientras que, en el 2018, la tasa esperada era de $54 \%$ y la alcanzada fue de $6 \%$ (48\% no logrado). Comparada las tasas reales de utilización entre los años evaluados (2017 y 2018), se refleja una disminución del 5\%, lo que revela el uso deficiente de la capacidad del centro. Ver figura 1.

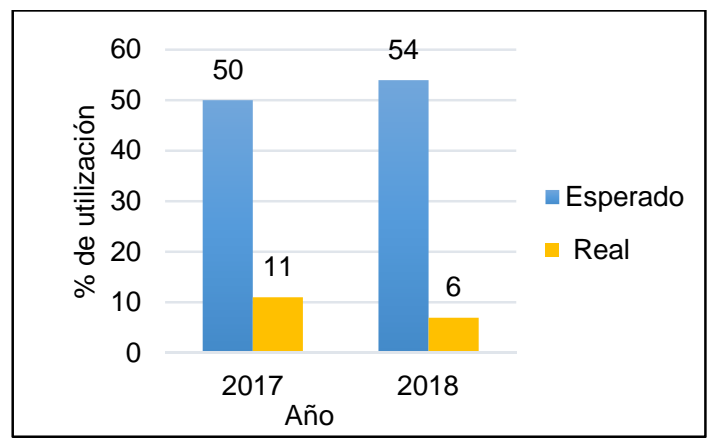

Figura 1. Tasa de utilización del CMPC esperada vs la real.

En relación con el número de productores beneficiados, para los años 2017 y 2018, solo 13 y 14 productores han utilizado el CMPC respectivamente [5], [6], de los 581 productores que se estimaba beneficiar. Lo que muestra claramente que solo un $2 \%$ de los productores esperados han aprovechado el beneficio del CMPC.

\subsubsection{Operaciones del CMPC}

En el centro se realizan dos operaciones principales: trasiego (cross dock) y procesamiento. Para ingresar los productos al CMPC, el productor debe estar registrado en la base de datos. Si no se encuentra registrado, el productor debe completar un formulario para su inscripción. Una vez inscrito, puede acceder a los beneficios de los diferentes servicios que el centro ofrece, con solo indicar cuál de ellos requiere.

El trasiego consiste en pasar el producto que traen los productores desde la finca, a los camiones de transportistas independientes y de allí a los centros de comercialización, como destino final. Este proceso incluye el pesaje del producto y la emisión de una etiqueta con toda la información relevante del producto y el productor. Sin embargo, este proceso ocasionalmente se cumple, debido a que los productores insisten en trabajar con un peso aproximado por caja o bolsa. Por otro lado, el camión que transporta los productos no siempre es refrigerado y, en su mayoría, se carga con diferentes productos que requieren de condiciones de temperatura y humedad distintas para su conservación. Otro aspecto relevante del proceso se refiere al hecho que cada productor debe efectuar el trasiego de su carga utilizando sus propios trabajadores, lo que podría causar deterioro del producto por la manipulación inadecuada.

Es importante destacar que el CMPC ofrece de manera gratuita el proceso de trasiego y aun cuando este no forma parte de los eslabones de la cadena de frío, es una práctica común realizada por los productores. Este accionar indujo a incluirla en los servicios brindados en el CMPC, con la intención de 
proveer un lugar físico adecuado para efectuar el proceso de trasiego, evitando que los productores utilicen malas prácticas que conduzcan a afectar la calidad e inocuidad del producto.

En cuanto al procesamiento del producto, este tiene la finalidad de mejorar la presentación y/o preservación del producto. Este proceso abarca una serie de operaciones, que van desde el preenfriamiento hasta el servicio de almacenaje, reempaque y transporte. Estas operaciones son: lavado, desterronado, cepillado, secado, clasificación, selección, empacado, etiquetado, encerado y pesado. Sin embargo, en la práctica, las operaciones descritas no siempre se llevan a cabo en su totalidad, porque depende de los requerimientos del productor y el tipo de producto. Esta fase llamada "procesamiento" tendría un precio al productor que dependería del tipo de rubro, peso, días de almacenamiento y otras variables de tratamiento dado al producto. Debido a la baja efectividad del CMPC, no se ha cumplido con lo planificado y no se han concretado los diferentes precios según las condiciones establecidas. Actualmente se ofrece de forma gratuita como una medida que busca incrementar el nivel de utilización del centro.

\subsection{Transporte refrigerado}

La columna vertebral de la cadena de frío es el transporte refrigerado, el cual hace posible que los productos puedan transportarse y distribuirse conservando su composición y calidad. Sin este elemento, la efectividad del sistema no se lograría.

A pesar que el transporte refrigerado es uno de los 3 componentes del proyecto, actualmente el servicio no se ofrece y el productor se hace responsable de todo el manejo del producto, en este aspecto. Son pocos los productores que cuentan con el transporte refrigerado, lo que se traduce en el rompimiento inmediato de la cadena de frío, ocasionando que las probabilidades de deterioro del producto sean más altas.

\subsection{Mercados públicos nacionales}

El último de los componentes del proyecto del Sistema Nacional de Cadena de Frío son los mercados públicos nacionales, cuya finalidad es proveer de alimentos a la población panameña, garantizando la inocuidad en el producto a través de prácticas de calidad y contribuyendo de esta manera a mejorar su calidad de vida de los mercados públicos que componen el proyecto, solo dos (2) se encuentran en funcionamiento: el mercado público de David y Merca Panamá.

Merca Panamá, tiene establecido claramente los requisitos para el ingreso de los productos, pero no contemplan aspectos relacionados a la calidad e inocuidad.

Cuando el productor comercializa sus productos en el mercado nacional, la negociación se realiza directamente con el arrendatario. Esto provoca la ausencia de un registro del producto ingresado, de la verificación de su calidad y de incentivos al productor que utilice el CMPC. Adicionalmente, se observa que los puestos de venta empleados para la comercialización del producto no reúnen las características para mantener las condiciones de temperatura y humedad requeridas para el producto durante su venta. Ver figura 2

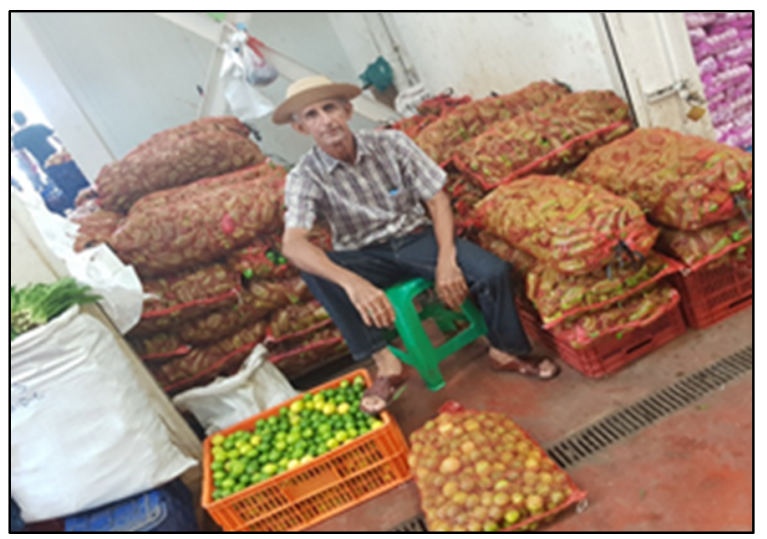

Figura 2. Puesto de venta en Merca Panamá, en donde se puede observar las formas de almacenamiento del producto para su venta.

\section{Fase 2: Resultados del análisis del sistema desde la perspectiva del productor de la región de Azuero}

\subsection{Caracterización de los productores de la región}

Con la intensión de poder establecer las estrategias más acertadas se realizó la caracterización y análisis de las prácticas de producción y comercialización de los productores de la región, la cual reflejó los siguientes resultados:

- En la región de Azuero solo el $0.74 \%$ de los productores encuestados siembra más de 6 hectáreas, lo que los ubica en la categoría de pequeños productores [7].

- De la muestra total, el $53 \%$ de los productores mantienen más de 15 años dedicados a la siembra, lo que señala una vasta experiencia, pero quizás con prácticas empíricas y muy aferradas que los lleva a resistirse a los cambios a favor de la implementación de nuevos métodos y tecnologías.

- Con respecto a la capacidad de planificación, el $61 \%$ de los productores no planifica su producción, lo que sustenta que la práctica es empírica. Respecto a los registros de siembra el $84 \%$ no lleva ningún tipo de documentación lo que dificultaría llevar la trazabilidad de los productos.

- El 54\% de los productores no tiene establecidos sus compradores, previo al periodo de siembra, del resto $46 \%$ (62 productores) que dijo tener comprador el $77 \%$ vende a la compañía Nestlé, Los Santos. Concluyendo que, en su mayoría, no guardan una relación sólida y/o permanente con sus clientes (compradores). La inestabilidad en la relación compra - venta puede ocasionar que parte o, incluso, la totalidad de la 
producción sea vendida a un precio muy por debajo del precio del mercado o, sencillamente, llegarse a perder.

- En el tema de comercialización, 76 de los encuestados venden a intermediarios, 59 realizan venta directa y 3 a cadena de supermercados. De los 59 que realizan venta directa, el $81 \%$ vende directamente a la Nestlé.

- En relación con las prácticas de transporte, de los 69 productores que dijeron ser responsables del transporte del producto, el $54 \%$ usa transporte alquilado, $42 \%$ utiliza transporte propio y $4 \%$, ambos. Ninguno de estos productores utiliza transporte refrigerado.

- En cuanto a la merma durante la comercialización, los productores aseguran que la merma se encuentra entre 0 y $25 \%$ del producto, con un costo de pérdida representado en el valor del producto.

\subsection{Análisis de la cadena de frío desde la perspectiva del productor}

Los resultados obtenidos de la encuesta acerca del nivel de conocimiento por parte del productor sobre el sistema de cadena de frío y su postura frente a este, reflejaron lo siguiente:

- El 98\% de los productores manifestó estar familiarizado con el concepto de cadena de frío y solo lo relacionan con el almacenamiento del producto.

- En cuanto a la existencia física del CMPC ubicado en El Ejido, 104 productores (77\%) dijo saber de su ubicación, pero de estos solo 8 lo han utilizado.

- Entre las principales razones por las cuales no utilizan el centro, señalaron que de: 44 productores dijeron no conocer el procedimiento y los beneficios, 37 manifestó no haber tenido la necesidad de utilizarlo y 34 productores dijeron que no sabían que el CMPC se encontraba funcionando. Se evidencia que la poca efectividad del CMPC se debe más que todo a la falta de divulgación de los beneficios que representa para el productor y de una orientación en cuanto a los requisitos para su utilización.

- De los 8 productores que han utilizado el centro, 3 productores aseguraron no volver a utilizarlo, por los siguientes motivos: que el peso total ingresado del producto al centro es mayor al peso del producto que sale, que una vez fuera del centro el producto se deteriora con mayor rapidez y que el sistema no contribuye con la venta del producto una vez ingresa al centro.

- Finalmente, se les consultó a aquellos productores que no han implementado la cadena de frío, si estarían interesados en utilizarla, a lo que el $87 \%$ dijo que sí. Mientras que los que no manifestaron interés argumentaron no encontrar beneficios para la venta del producto, sumado a que, los bajos volúmenes de producción no justifican su uso.

\section{Diagnóstico}

Para determinar puntualmente los problemas, las causas y sus efectos en la cadena de frío, y establecer las estrategias adecuadas, se realizó el diagnóstico de cada uno de los elementos analizados.

\subsection{Usuario principal (productor)}

El bajo nivel de conocimiento sobre los requerimientos que exige el sistema para su adecuado uso y los potenciales beneficios que de él se derivan, han sido uno de los principales obstáculos a los que los productores se han enfrentado en la implementación efectiva del sistema de la cadena frío.

Si listamos aquellos factores clave que han incidido a que el sistema no represente un punto de interés para el productor, observamos una postura - digamos algo conservadora-, que impide a este el crecimiento y la apertura a nuevas oportunidades. Esta panorámica nos lleva a pensar que si se logra que el productor se adapte y confíe en las bondades que ofrece la cadena de frío, en esa medida, él logrará cambios en sus prácticas de producción que lo llevarán a incrementar los volúmenes de producción, por ende, sus beneficios y/o utilidades. Veamos, específicamente, esas debilidades:

- La existencia predominante de pequeñas parcelas impide en el productor el crecimiento de sus volúmenes de producción. Los bajos volúmenes de producción se traducen en una limitante para que el productor tenga acceso a programas, proyectos, asistencia y capacitación técnica, entre otros beneficios que le permitan incursionar en nuevos segmentos de mercado.

- Los bajos volúmenes de producción disminuyen el poder para aprovechar las economías de escala.

- Los limitados niveles de producción generan incertidumbre en el productor con relación a la rentabilidad de la inversión, lo que significa para ellos, costos más altos y ninguna posibilidad de beneficios.

- La poca capacidad de gestión del productor disminuye su poder de negociación y dificulta la implementación de prácticas adecuadas para el manejo post cosecha y del sistema de la cadena de frío.

- Para ambos, la falta de planificación y el bajo volumen de producción llevan al productor a utilizar intermediarios. Esto trae consigo, el aumento en el precio para el consumidor final y la disminución del ingreso por venta del productor, adicional a un desinterés para dar un valor agregado a su producto que contribuya a una mayor rentabilidad en su inversión. Cada uno de los factores señalados afecta directamente al primer eslabón del sistema, el CMPC.

\subsection{El Centro de Manejo Post Cosecha}

Opera con una tasa de utilización real por debajo de la tasa esperada, lo que confirma los elementos causantes señalados anteriormente y que se resumen principalmente en la falta de 
conocimiento, y de capacidad de gestión por parte de los productores, para administrar los recursos disponibles a favor de la producción. Se agregan a estos factores, las deficiencias del centro de manejo post cosecha para establecer políticas formales de pago que generen confianza en el productor y el establecimiento de políticas de incentivos que fomenten el uso del CMPC.

\subsection{Transporte refrigerado}

La oferta de transporte refrigerado en la región es prácticamente nula. A pesar de uno de los componentes del sistema, este servicio no es ofrecido y es una de las razones por la cual el productor no implementa la cadena de frío.

\subsection{Mercados públicos nacionales}

No cuentan con políticas o requisitos para el ingreso de los productos pertenecientes a la red de frío, por lo que no asegura la calidad e inocuidad ni obliga a mantener la cadena de frío. La ausencia de estas políticas no contribuye a incentivar en el productor la implementación efectiva de la cadena.

Con relación a los puestos de venta, la cadena de frío carece de la totalidad de los equipos requeridos para cumplir con el proceso hasta la compra por el consumidor y se obtenga así los beneficios que la cadena aporta.

\section{Estrategias}

Podemos tomar en cuenta los resultados obtenidos de los análisis realizados, así como proyectos llevados a cabo por países como México, quien desarrolló un programa nacional de agrologística, en el año 2014, con un horizonte de cumplimiento de 30 años. En este programa establecieron una serie de políticas públicas con la finalidad de convertirse en unos de los países líderes en la exportación de productos hortofrutícolas [8]. Por otro lado, el análisis para el desarrollo de la cadena de frío en países de África [1] y en la India [9]; indica que se diseñaron las estrategias que orientarán a los entes competentes en el manejo del sistema de la cadena de frío con la finalidad de incrementar su nivel de utilización y encaminarla hacia el logro de los objetivos para los que fue implementada.

Las estrategias que definen las posibles acciones de fortalecimiento y mejoras al sistema se construyeron tomando en cuenta los Planes del Gobierno Nacional (2019 - 2024). De esta forma se alinearán y contribuirán, de una u otra manera, con las políticas de desarrollo y crecimiento económico del país.

En la figura 3, se muestran las estrategias y en la tabla 2, se detallan las acciones, recursos y responsables de ejecutarlas. Se sugieren como responsables los descritos en la tabla; sin embargo, estos entes podrían cambiar dependiendo de los requerimientos de la acción, al igual que los recursos señalados. Cabe resaltar, que estas estrategias solo son la base para el desarrollo de un plan de acción, que requiere de la participación de todos los entes involucrados en materia, razón por la que no incluye el análisis de tiempo y costo.

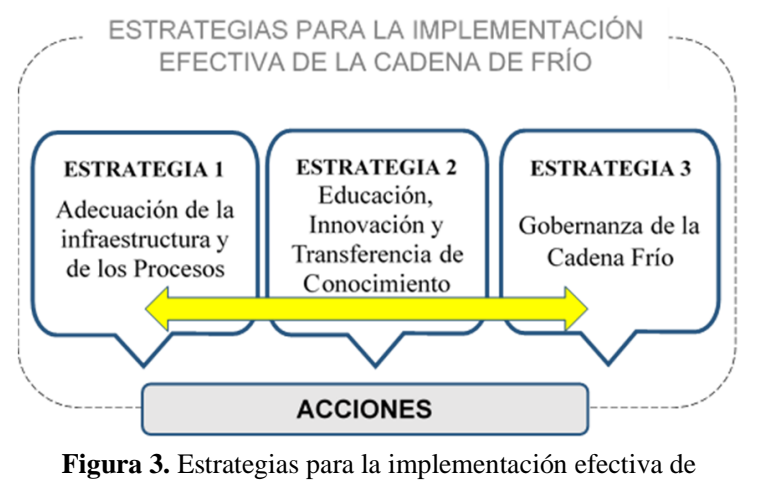
la cadena de frío.

Tabla 2. Objetivos, estrategias y líneas de acción propuestas E-1. Adecuación de la infraestructura y de los procesos

Objetivo: Adecuar la infraestructura física y de los procesos que integran el sistema actual de cadena de frío.

\begin{tabular}{|c|c|}
\hline Acciones & Respo \\
\hline $\begin{array}{l}\text { Establecer políticas de precio por la } \\
\text { utilización de los servicios que oferta el } \\
\text { centro de post cosecha según rubro, } \\
\text { volumen a ingresar, tipo de proceso y } \\
\text { días de almacenamiento }\end{array}$ & $\begin{array}{l}\text { Mercados } \\
\text { Nacionales de } \\
\text { Cadena de Frío, } \\
\text { S.A. }\end{array}$ \\
\hline $\begin{array}{l}\text { Certificar los procesos que se realizan } \\
\text { en el CMPC mediante la norma Cool } \\
\text { Chain Quality Indicators, de manera } \\
\text { que se asegure la calidad de las } \\
\text { operaciones realizadas. }\end{array}$ & $\begin{array}{l}\text { Mercados } \\
\text { Nacionales de } \\
\text { Cadena de Frío, } \\
\text { S.A. }\end{array}$ \\
\hline $\begin{array}{l}\text { Establecer el protocolo específico para } \\
\text { el procesamiento o almacenamiento de } \\
\text { cada rubro en los CMPC. }\end{array}$ & $\begin{array}{l}\text { Mercados } \\
\text { Nacionales de } \\
\text { Cadena de Frío, } \\
\text { S.A. }\end{array}$ \\
\hline $\begin{array}{l}\text { Proporcionar una } \\
\text { productos que ingre } \\
\text { garantice la inocuid }\end{array}$ & $\begin{array}{l}\text { Mercados } \\
\text { Nacionales de } \\
\text { Cadena de Frío, } \\
\text { S.A. }\end{array}$ \\
\hline $\begin{array}{l}\text { Establecer mecanismos de enlace y } \\
\text { diálogo permanente con las cadenas de } \\
\text { supermercados, industrias u otros } \\
\text { mercados, para consolidar una relación } \\
\text { de respeto y eficaz con el productor y } \\
\text { estas entidades }\end{array}$ & $\begin{array}{l}\text { Mercados } \\
\text { Nacionales de } \\
\text { Cadena de Frío, } \\
\text { S.A. } \\
\text { IMA }\end{array}$ \\
\hline $\begin{array}{l}\text { Dotar al sistema nacional de la cadena } \\
\text { de frío de los servicios de transporte } \\
\text { refrigerado. }\end{array}$ & $\begin{array}{l}\text { Mercados } \\
\text { Nacionales de } \\
\text { Cadena de Frío, } \\
\text { S.A. }\end{array}$ \\
\hline $\begin{array}{l}\text { a los pues } \\
\text { s necesarios }\end{array}$ & $\begin{array}{c}\text { Mercados } \\
\text { Nacionales de }\end{array}$ \\
\hline
\end{tabular}




\begin{tabular}{|c|c|}
\hline $\begin{array}{l}\text { los productos procurando mantener las } \\
\text { condiciones de temperatura y humedad. }\end{array}$ & $\begin{array}{l}\text { Cadena de Frío, } \\
\text { S.A. }\end{array}$ \\
\hline $\begin{array}{l}\text { Establecer como requisito para ingresar } \\
\text { a los mercados públicos nacionales, la } \\
\text { certificación por el CMPC para todos } \\
\text { los productos que pertenecen a la red de } \\
\text { frío. }\end{array}$ & $\begin{array}{c}\text { Mercados } \\
\text { Nacionales de } \\
\text { Cadena de Frío, } \\
\text { S.A. }\end{array}$ \\
\hline $\begin{array}{l}\text { Crear un departamento que garantice el } \\
\text { cumplimiento del reglamento de los } \\
\text { productos que ingresan a los MPN, a } \\
\text { través de las inspecciones a los puestos } \\
\text { de venta. }\end{array}$ & $\begin{array}{c}\text { Mercados } \\
\text { Nacionales de } \\
\text { Cadena de Frío, } \\
\text { S.A. }\end{array}$ \\
\hline \multirow{2}{*}{\multicolumn{2}{|c|}{$\begin{array}{l}\text { E-2. Innovación, educación y transferencia de } \\
\text { conocimiento } \\
\text { Objetivo: Priorizar la inversión para la modernización del } \\
\text { sistema de la cadena de frío articulando la inversión técnica } \\
\text { y de capital humano para lograr ventaja competitiva en el } \\
\text { sector agrícola. }\end{array}$}} \\
\hline & \\
\hline Acciones & Responsables \\
\hline $\begin{array}{l}\text { Diseñar e implementar programas de } \\
\text { divulgación y promoción de la } \\
\text { importancia de utilizar el sistema de la } \\
\text { cadena de frío y de los beneficios que se } \\
\text { generan de su implementación. }\end{array}$ & $\begin{array}{c}\text { Mercados } \\
\text { Nacionales de } \\
\text { Cadena de Frío, } \\
\text { S.A. MIDA }\end{array}$ \\
\hline $\begin{array}{l}\text { Diseñar e implementar programas de } \\
\text { capacitación y de formación para los } \\
\text { usuarios del sistema (productor, } \\
\text { extensionista y otros), en temas de } \\
\text { manejo de productos antes, durante y } \\
\text { después de la cosecha, inocuidad, } \\
\text { calidad, y trazabilidad del producto, así } \\
\text { como, de aspectos relacionados con la } \\
\text { producción y la visión empresarial. }\end{array}$ & MII \\
\hline $\begin{array}{l}\text { Desarrollar aplicaciones que permitan } \\
\text { el acceso a información y/o datos } \\
\text { relativos al mercado, permitiendo un } \\
\text { seguimiento a los distintos procesos } \\
\text { dentro de la cadena de frío, en cuanto a } \\
\text { la planificación de la demanda, tiempos } \\
\text { de entrega y otros parámetros de interés. }\end{array}$ & $\begin{array}{c}\text { Mercados } \\
\text { Nacionales de } \\
\text { Cadena de Frío, } \\
\text { S.A. } \\
\text { MIDA } \\
\text { IMA } \\
\text { IDIAP }\end{array}$ \\
\hline $\begin{array}{l}\text { Diseñar, promover y construir acuerdos } \\
\text { de colaboración con las universidades } \\
\text { que deriven en proyectos para impulsar } \\
\text { desde la docencia, la investigación y } \\
\text { extensión, la temática de la cadena de } \\
\text { frío. }\end{array}$ & $\begin{array}{l}\text { Mercados } \\
\text { Nacionales de } \\
\text { Cadena de Frío, } \\
\text { S.A. MIDA } \\
\text { IDIAP } \\
\text { universidades } \\
\text { oficiales y } \\
\text { particulares }\end{array}$ \\
\hline
\end{tabular}

\section{E-3. Gobernanza de la cadena de frío}

Objetivo: Impulsar la implementación efectiva de la cadena de frío.

\begin{tabular}{|c|c|}
\hline Acciones & Responsables \\
\hline Creación de un marco de gobernanza &
\end{tabular}

que permita la coordinación interinstitucional necesaria para el desarrollo de las políticas y programas que generen oportunidades y soluciones al productor, garantizando el éxito en el uso de la cadena de frío.

Promover el desarrollo de capacidades institucionales y modelos de gestión para lograr una administración efectiva del sistema nacional de la cadena de frío.

Nacionales de

Cadena de Frío

S.A., IMA MIDA

\begin{tabular}{|l|c|}
\hline $\begin{array}{l}\text { Instituir normas y reglamentos de } \\
\text { gestión de la calidad e inocuidad de los }\end{array}$ & MIDA \\
$\begin{array}{l}\text { alimentos, que aseguren un producto de } \\
\text { calidad y se garantice su cumplimiento. }\end{array}$ & DEPA \\
\hline (MINSA)
\end{tabular}

Establecer políticas que alienten la creación de grupos o modelos de asociaciones de pequeños y medianos productores que contribuyan en la generación de economías de escala con un mayor valor agregado

Crear mecanismos de apoyo a los productores mediante la implementación de incentivos que promuevan la utilización de la cadena de frío, en especial aquellos que guardan relación con la compra institucional de los productos certificados por la cadena para atender los programas de alimentación escolar.

\begin{tabular}{|c|c|}
\hline $\begin{array}{l}\text { Garantizar un precio } \text { justo y } \\
\text { competitivo para los productos } \\
\text { certificados por la cadena de frío. }\end{array}$ & $\begin{array}{c}\text { MIDA } \\
\text { IMA }\end{array}$ \\
\hline
\end{tabular}

\section{Conclusiones}

El análisis y diagnóstico realizado proyectó resultados muy interesantes que avalan la falta de estrategias para la implementación efectiva del Sistema de la Cadena de Frío en Panamá. Se resaltan como aspectos concluyentes de la investigación y que permitieron el diseño de las estrategias:

- Las expectativas en la cantidad de productos que manejaría la capacidad instalada del centro de manejo post cosecha desde su implementación no ha cumplido con los objetivos trazados. Solo el $8 \%$ de los productores encuestados ha utilizado el centro, 
sustentado en el hecho de la falta de conocimiento que se tiene sobre este. En particular revela una deficiencia en la capacidad de gestión por parte de los entes competentes en el manejo del CMPC.

- Los datos recabados revelan que un alto porcentaje de productores muestra interés en conocer los beneficios que aporta la implementación del sistema de la cadena de frío y evaluar las posibilidades de poder utilizarlo.

- Se debe tener en cuenta que en la región se ubican pequeños productores, caracterizados por la debilidad en su capacidad de gestión y planificación, por lo que es mejor trabajar en estas capacidades, sería un punto a favor de la implementación de los nuevos métodos y tecnologías.

- Solo 59 de los productores encuestados realizan la venta de sus productos de forma directa y 3 a cadena de supermercados, revelando que urgen políticas $o$ programas que ayuden al productor en la comercialización del producto y que lo incentiven a utilizar la cadena de frío.

- En cuanto al sistema de cadena de frío, es necesario dotarlo con los equipos necesarios para el transporte y la conservación de los productos en los mercados.

- Se carece de la coordinación entre las instituciones relacionadas al sector agro, lo que impide el desarrollo de programas y políticas, así como la escasa participación del sector privado.

\section{Recomendaciones}

- Encargar a las autoridades competentes de los Mercados Nacionales de la Cadena de Frío S.A., atender las acciones planteadas.

- Diseñar e implementar programas de promoción y difusión -intensiva y permanente-, para dar a conocer a los productores de la región de Azuero, acerca de la importancia en el uso del sistema integral de la cadena de frío y de cada uno de los beneficios y servicios que ofrece.

- Llevar a cabo políticas públicas que eliminen los obstáculos que limitan al productor a ser más competitivo y que se orienten a fortalecer su capacidad de gestión y a mejorar sus prácticas de producción.

- Los Mercados Nacionales de Cadena de Frío S.A., dotar al sistema con el transporte refrigerado y realizar las evaluaciones en cuanto a costo, cantidad de vehículos, rutas, políticas de uso, de forma que se facilite la prestación del servicio a los productores.

- Incentivar entre todos los actores de la actividad económica el uso eficiente de los recursos productivos y del sistema de cadena de frío.

- Involucrar a las instituciones relacionadas con el sector agropecuario, así como las empresas privadas, en el desarrollo de programas y políticas que impulsen el uso adecuado de la cadena de frío.

\section{Trabajo futuro}

Con la finalidad de ampliar la investigación y contribuir al desarrollo y ejecución de las acciones propuestas se resaltan algunas líneas de trabajo futuro. Cabe resaltar, que para el desarrollo de cada una de las acciones se requiere de un estudio previo del entorno actual.

- Desarrollar un programa para la alineación de las entidades involucradas en materia, en donde se evalué, defina y detalle el rol de cada una de ellas en relación con el desarrollo del sistema de cadena de frío.

- Evaluar las políticas de acceso a los mercados, así como los canales de comercialización, a fin proponer mejoras o políticas que facilite al productor el acceso a los mercados.

- Analizar los programas de incentivo y ayuda al productor. Así como evaluar, los programas de promoción y capacitación de los usuarios de la cadena de frío, una vez existan. De no existir, proponer programas y realizar proyectos piloto para evaluar su efectividad. Esto con la finalidad de proponer mejoras y/o nuevos programas que motiven al productor a implementar el sistema de cadena de frío.

- Analizar la demanda de transporte refrigerado, que permita dotar al sistema con las cantidades y tipos de transporte requerido, así como evaluar las políticas de uso (por ejemplo, días de transporte según rubros, costo de uso, rutas, etc.).

\section{AGRADECIMIENTOS}

Extiendo mi más sincero agradecimiento a los jefes y colaboradores de las agencias del MIDA de: Chitré, Pesé, Parita, Santa María, Guararé, Macaracas, Tonosí, Tres Quebradas y Los Santos por el apoyo, y tiempo dedicado. A los productores de la región de Azuero, razón de este estudio, por su tiempo y disposición. A los profesores Waldo Moreno, Celinda Pérez y Bolívar Bernal M. por ser guía en el desarrollo de esta investigación.

\section{REFERENCIAS}

[1] J. S. Elhadi Yahia Kazuz, «Developing the Cold Chain for Agriculture in the Near East and North Africa» 2015. [En línea]. Available: http://www.fao.org/3/a-ax746e.pdf.

[2] C. P. A. Calixto Mendoza, Manual práctico para la gestión logística, España: Universidad Del Norte, Edisiones Uninorte, (2015).

[3] ProColombia, Logística de perecederos y cadena de frío en Colombia, 2014.

[4] Consocio Panamá Frío, «Diseño, Construcción, Equipamiento y Puesta en Marcha del Sistema Logístico de Cadena de Frío para la República de Panamá,» Panamá, 2012. 
[5] Mercados Nacionales de la Cadena de Frío, S.A., «Memoria MNCF,» 2017. [En línea]. Available: https://www.cadenadefrio.com.pa/content/transparencia/MEM ORIA-2017--MNCF.pdf.

[6] Mercados Nacionales de la Cadena de Frío, S.A., «Memoria MNCF,» 2018. [En línea]. Available: https://www.cadenadefrio.com.pa/content/transparencia/MEM ORIA-2018-Final.pdf.

[7] O. d. 1. N. U. p. 1. A. y. 1. A. (FAO)., «Agricultores Pequeños y Familiares. Obtenido de,,» 2013. [En línea]. Available: http://www.fao.org/3/ar588s/ar588s.pdf.

[8] G. D. R. P. y. A. (. Secretaría de Agricultura, «Programa Nacional de Agrologística México,» 2015. [En línea]. Available: https://nccd.gov.in/PDF/ChallengeColdChainDevelopment.pdf.

[9] Y. Bank, «Cold Chain Opportunities in India» 2018. [En línea]. Available:

https://www.yesbank.in/pdf/cold_chain_opportunities_in_indi a.pdf.

[10] Y. Valdés, «Perdidas en el Mercado por Merma,» 17 Marzo 2018. [En línea]. Available: https://www.panamaamerica.com.pa/economia/136-millonesen-perdidas-en-el-mercado-por-mermas-1100286.

[11] E. N. f. R. Development, «Smart agri-food supply chains» 2016. [En línea]. Available: https://enrd.ec.europa.eu/sites/enrd/files/s1_scra_factsheetfinal.pdf. 